\title{
Wie es Euch gefällt
}

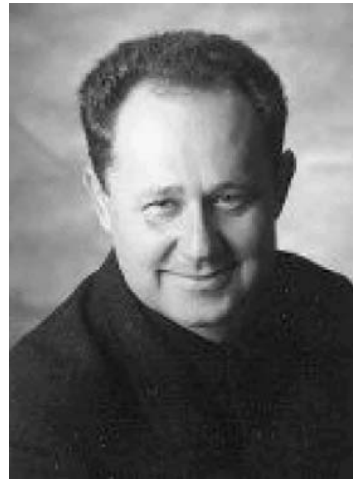

Prof. Dr. Manfred Wildner

\section{Bibliografie}

DOI http://dx.doi.org/ 10.1055/s-0030-1249061 Gesundheitswesen 2010; 72: 257-258

(c) Georg Thieme Verlag KG Stuttgart · New York ISSN 0941-3790

Korrespondenzadresse Prof. Dr. M. Wildner Bayerisches Landesamt für Gesundheit und Lebensmittelsicherheit Veterinärstraße 2 85762 Oberschleißheim manfred.wildner@|gl.bayern.de
William Shakespeare hat uns mit dem im Jahr 1600 erschienen Schauspiel „Wie es Euch gefällt“ ein munteres Stück Hirten-Dichtung hinterlassen. Die Handlung: „Herzog Friedrich entmachtet seinen älteren Bruder Herzog Senior, der daraufhin mit einer Anzahl treuer Lords in den Ardenner Wald in die Verbannung geht. Rosalind, die älteste Tochter Seniors, bleibt an Fredericks Hof bei dessen mit ihr befreundeten Tochter Celia. Nach dem Tod von Sir Rowland de Bois wird sein ältester Sohn Oliver Haupterbe, sein jüngster Sohn Orlando erhält nur tausend Kronen“ [1]. Können Sie noch folgen? Um es kurz zu machen: König Senior war eine Art „Aussteiger“. Im Wald, sprich in der unverfälschten Natur, liegt die Rettung. Alle, die ihm dorthin folgen, führen ein glückliches (Liebes-)Leben in fröhlicher Kommunität und größter Freiheit. Zu guter Letzt bekehrt sich sogar der verräterische Herzog Frederick durch die Begegnung mit einem Einsiedler und gibt Herzog Senior sein Herzogtum zurück [2]. Alles wird gut.

Derartige archetypischen Vorstellungen von der Aktivierung der Selbstheilungskräfte durch die Natur sind vermutlich so alt wie die Menschheit - zumindest seit die ersten Exemplare des modernen Menschen aufgerichtet den Wald verließen. Explizite Heilungserwartungen sind auch nicht auf den westlichen Kulturkreis beschränkt. Der Journalist Tiziano Terzani, selbst an Krebs erkrankt, gibt in seinem autobiografischen Bericht „Noch eine Runde auf dem Karussell“ einen nachdenklichen Einblick in den Megamarkt esoterischer Heilungsangebote und Heilungsversprechen weltweit - von Boston bis Bagdad, von Kathmandu bis Kalifornien [3]. Haben manche alternativen Heilverfahren nicht auch ihre nachweisbare Wirksamkeit? Und sei es die Aktivierung von körperimmanenten Heilvorgängen, wie sie mit dem Begriff des Placebos verbunden werden? Ein Anlass, sich mit Placebo-Vorgängen etwas näher auseinander zu setzen.

„Wie es Euch gefällt...“ - Placebo bedeutet in der wörtlichen Übersetzung aus dem Lateinischen „Ich werde gefallen“. Es ist eine Anspielung an den Psalm 114, Vers 1-9 der lateinischen Vulgata-Übersetzung der Bibel: „Placebo Domino in regione vivorum - Ich werde dem Herrn gefallen im Lande der Lebenden“. Dieser Vers war Bestandteil der mittelalterlichen Totenliturgie. Die religiöse Bedeutung wandelte sich in eine weltliche im England des 14. Jahrhunderts: Der Ausdruck „to sing a placebo“, eine Anspielung auf die bezahlten Gesänge am Grab, entwickelte sich zum Synonym zu „schmeicheln“ [4]. Ausgangspunkt der moder- nen Placebo-Forschung waren Publikationen insbesondere über die Wirksamkeit der verdeckten Gabe von einfacher Kochsalzlösung an Stelle von Morphin bei Schmerzpatienten und auch bei anderen Krankheitsbildern. Henry K. Beecher berichtete über diese Erfahrungen mit der Macht des Placebos an Hand von ausgewählten eigenen und fremden Studien im Jahr 1955 („The powerful placebo“, [5]). Angeblich ging sein Interesse auf eigene Erfahrungen im zweiten Weltkrieg zurück, als ihm die Medikamente für die Behandlung Kriegsverletzter an der italienischen Front ausgingen [4]. Von Levine konnte 1979 nachgewiesen werden, dass der schmerzlindernde Effekt von Placebos durch Opiatantagonisten unterdrückt werden kann: ein deutlicher Hinweis auf die biochemische Wirksamkeit der Placebovermittelten Schmerzunterdrückung [6]. Objektive Belege für die Wirksamkeit zur Schmerzlinderung zeigen sich auch in der Positronen-Emissionen-Tomografie (PET-Scanner) [7].

Obwohl an einzelnen Patienten Placeboreaktion nachweisbar sind, wird die klinische Relevanz des statistisch gemessenen Placeboeffektes in kontrollierten Studien kritisch bewertet: nur kleine Effekte waren in einer sorgfältig durchgeführten Metaanalyse nachweisbar [8]. Eine andere Arbeitsgruppe berichtet am Beispiel des Colon irritabile von deutlicheren Effekten mit kombinierbarer Wirksamkeit der Komponenten aufmerksame Anamneseerhebung, therapeutisches Ritual (Placebo in engerem Sinn) und unterstützende Therapeuten-Patienten-Beziehung. Dabei kommt der Therapeuten-Patienten-Interaktion die größte Bedeutung zu [9]. Auch im Zusammenhang mit Akupunktur gegen Kopfschmerz ist zweifelhaft, ob die nachweisbaren Effekte auf die Akupunktur an sich zurückzuführen sind: zumindest bei Migraine hat eine Akupunktur an unspezifischen Hautstellen die gleiche Wirksamkeit wie eine Akupunktur an den klassischen Akupunkturpunkten [10,11].

Einer daraus abgeleiteten Empfehlung zum Einsatz von Akupunktur, welche als echte oder unspezifische Akupunktur wirksamer und gleichzeitig nebenwirkungsärmer als eine medikamentöse Therapie sein soll, kann nur mit kritischer Distanz gefolgt werden. Vor dem Hintergrund aktueller Placeboforschung sollte vor allem auf die Qualität der Arzt-Patienten-Beziehung fokussiert werden $[9,12]$. Hauptsächliche Wirkungsmechanismen sind bedingte Reflexe, Erwartungshaltungen und Bedeutungszuschreibungen des Patienten sowie die Applikationsform [4,1214]. Ein begleitender Pfad der Effektvermittlung 
ist der „healthy adherer“-Effekt bei guter therapeutischer Compliance [15]. Alternative Erklärungen zum Placeboeffekt sind günstige Spontanverläufe und ein statistischer Effekt des „regression to the mean“, d.h. der unwahrscheinlichen Wiederholung einmaliger extremer Ausgangswerte der Studienteilnehmer $[4,12]$.

Die Relativierung und Differenzierung des Placeboeffektes tut dem Wert des Placebos im Rahmen Placebo-kontrollierter klinischer Studien keinen Abbruch. Die wohl älteste dokumentierte Episode der Verwendung einer Placebokontrolle ist mit dem amerikanischen Naturwissenschaftler und Staatsmann Benjamin Franklin (1706-1790) verbunden. Er war vom französischen König zum Mitglied eines Komitees berufen worden, welches die Behauptungen Franz Anton Mesmers prüfen sollte. Mesmer gab an, ein körperliches Fluidum aus der Distanz beeinflussen zu können. Franklins Versuchsaufbau bestand in der Trennung der Studienteilnehmerinnen von dem Ausführenden des Mesmerismus. Die Anwesenheit oder Abwesenheit dieser Person war durch einen Vorhang verborgen, wurde von den Versuchspersonen jedoch angenommen. Er konnte nachweisen, dass der Erfolg des Mesmerismus lediglich von der Erwartungshaltung der Versuchspersonen abhing. Dies widerlegte die behauptete Wirksamkeit der Methode an sich.

Das vorliegende Heft unserer Zeitschrift beschäftigt sich in bester Franklinscher Tradition mit der vorurteilsfreien wissenschaftlichen Bewertung von Fragen wie der Zufriedenheit von Patienten mit der ärztlichen Dienstleistung, Distanzen zu Krankenhäusern und mindestmengenrelevanten Eingriffen, dem Beziehungsmanagement zwischen Krankenhaus und Krankenkasse, der Überführung neuer Konzepte in die Regelversorgung, der psychosozialen Gesundheit bei Kindern und Jugendlichen, regionaler gesundheitlicher Ungleichheit, Internet-Inhalten und telefonischem Fall-Management.

Übrigens: auch Placebos haben Risiken und Nebenwirkungen. Zum einen wird von einem schädlichen „Nocebo“-Effekt in Analogie zum heilenden „Placebo“-Effekt berichtet. Mindestens so bedeutsam ist jedoch, dass bei einer Placebogabe unter Umständen eine Standardtherapie vorenthalten wird. Die Deklaration von Helsinki der World Medical Association legt in ihrer aktuellen Überarbeitung von 2008 in Paragraph 23 fest, das Nutzen, Risiken, Lasten und Wirksamkeit einer neuen Intervention gegenüber der jeweils besten gegenwärtigen bewährten Intervention zu prüfen sind. Nur als Ausnahme zu dieser Grundregel ist die Verwendung von Placebo ethisch akzeptabel: Dann nämlich, wenn solche bewährten Verfahren nicht bestehen oder wenn zwingende methodische Gründe dies erfordern und die Probanden im therapiefreien bzw. Placebo-Arm der Studie dadurch keinen ernsten Risiken ausgesetzt sind. Übertragen auf die Anwendung von neuen Verfahren im bunten Bereich der alternativen Heilverfahren darf dies auch als Warnung gegenüber einem allzu sorglosen „Wie es Euch gefällt“ verstanden sein. Und auch gegenüber einer Selbstimmunisierung alternativer Verfahren gegen wissenschaftliche Kritik: dann nämlich, wenn für deren Prüfung andere Gesetze der Wissenschaftlichkeit gelten sollen.

Shakespeare starb im Alter von 52 Jahren. Auf seinem Grab in der Heiligen Dreifaltigkeitskirche in Stratford-upon-Avon, ist folgende Inschrift in die Steinplatte eingemeißelt: Good frend for Jesus sake forbeare,/to digg the dust enclosed heare./bleste be the man that spares thes stones,/and curst be he that moves my bones (dt. Guter Freund, im Namen Jesu grabe nicht/den Staub auf, der hier eingeschlossen ist./Gesegnet sei derjenige, der diese Steine achtet,/und verflucht der, der meine Gebeine bewegt). Im Sinne einer aufgeklärten Evidenz-basierten Medizin ist allerdings zu erwarten, dass die vielfältigen angebotenen Versorgungsformen - im Gegensatz zu einem „Wie es Euch gefällt“ - bei entsprechender Evidenzlage doch in ihren Gebeinen bewegt werden: Was heute gilt, kann morgen falsch sein oder zumindest anders einzuordnen sein. Wissenschaft ist nie abgeschlossen - sie ist eben, bei aller Unterhaltsamkeit, keine Belletristik.

\section{Literatur}

1 Wikipedia. Wie es Euch gefällt. URL: http://de.wikipedia.org/wiki/ Wie_es_euch_gefällt Zugriff am 01.01.2010

2 William Shakespeare: "As You Like It". Englisch-Deutsche Studienausgabe. Deutsche Prosafassung und Anmerkungen von Ilse Leisi, Einleitung und Kommentar von Hugo Schwaller. Stauffenburg, Tübingen; 2000

3 Terzani T. Noch eine Runde auf dem Karussell: Vom Leben und Sterben. Knaur, München; 2007

4 Schönbächler G. Placebo. Schweiz Med Forum 2007; 7: 205-210

5 Beecher K. The powerful placebo. J Am Med Ass 1955; 159: 1602-1606

6 Levine JD, Gordon NC, Bornstein JC et al. Role of pain in placebo analgesia. Nat Acad Sci USA 1979; 76: 3528-3531

7 Petrovic P, Kalso E, Peterson KM et al. Placebo and opioid analgesia imaging a shared neuronal network. Science 2002; 295: 1737-1740

8 Hrobjartsson A, Gotzsche PC. Is the placebo effect powerless? An analysis of clinical trials comparing placebo with no treatment. N Engl J Med 2001; 344: 1594-1602

9 Kaptchuk TJ, Kelley JM, Conboy LA et al. Components of placebo effect: randomized controlled trial in patients with irritable bowel syndrome. Br Med J 2008; 336 (7651): 999-1003

10 Linde $K$, Allais G, Brinkhaus B et al. Acupuncture for tension-type headache. Cochrane Database Syst Rev 2009 Jan 21 (1): CD007587

11 Linde $K$, Allais G, Brinkhaus B et al. Acupuncture for migraine prophylaxis. Cochrane Database Syst Rev 2009 Jan 21 (1): CD001218

12 Breidert M, Hofbauer K. Placebo: Mißverständnisse und Vorurteile. Dt Ärzteblatt 2009; 106 (46): 751-755

13 Barker Bausell R, Lao L, Bergman $S$ et al. Is acupuncture analgesia an expectancy effect? Preliminary evidence based in participants' perceived assignments in two placebo-controlled trials. Eval Health Prof 2005; 28: 9-26

14 Linde K, Witt CM, Streng A et al. The impact of patient expectation on outcomes in four randomized controlled trials of acupuncture in patients with chronic pain. Pain 2007; 128: 264-271

15 Simpson SH, Eurich DT, Majumdar SR et al. A meta-analysis of the association between adherence to drug therapy and mortality. Br Med J 2006; 333 (7557): 15 Epub 2006 Jun 21 